\title{
Atellica CH 930 chemistry analyzer versus Cobas 6000 c501 and Architect ci4100 - a multi-analyte method comparison
}

\author{
Imola Györfi”, Krisztina Pál", Ion Bogdan Mănescu*, Oana R. Oprea, Minodora \\ Dobreanu
}

Clinical Laboratory, County Emergency Clinical Hospital of Targu Mures, Romania, Dept Laboratory Medicine, George Emil Palade University of Medicine, Pharmacy, Science, and Technology of Targu

Mures, Romania

\begin{abstract}
Large clinical laboratories often rely on multiple chemistry analyzers. However, when a new analyzer is introduced, the laboratory must establish whether the old and new methods are comparable and can be used interchangeably. In this study, we compared the newly introduced Atellica CH930 chemistry analyzer with the already established Architect ci4100 and Cobas 6000 c501 from our laboratory.

Patient samples were randomly selected from daily routine testing and a total of 22 analytes were investigated. Total error $\left(T E_{o b s}\right.$ ) between test (Atellica) and comparative (Architect and Cobas) methods was calculated at relevant medical decision levels (MDL). For demonstrative purposes, the assessment of method comparability was based on three different criteria: allowable total error (TE ) derived from biological variation (BV), CLIA proficiency testing criteria for acceptable analytical performance, and CLIA-calculated Sigma metrics. These sets of analytical performance specifications were also compared, and their strengths and limitations are discussed in this paper.

Performance of Atellica CH930 against Architect ci4100 was acceptable or nearly acceptable at 82\%, 95\%, and $64 \%$ of the 22 investigated MDLs across 9 analytes, according to BV-TE ${ }_{a}, C L I A-T E_{a}$, and CLIA-calculated Sigma metrics, respectively. Similarly, performance of Atellica CH930 against Cobas 6000 c501 was acceptable or nearly acceptable at $61 \%, 93 \%$, and $63 \%$ of the 54 investigated MDLs across 22 analytes, according to BV-TE, CLIA$T E_{a}$, and CLIA-calculated Sigma metrics, respectively. However, method comparability should not be evaluated by a "one size fits all" approach as some analytes require different criteria of acceptability, ideally based on medically allowable error and clinical outcome.
\end{abstract}

Received: $21^{\text {st }}$ July 2021; Accepted: 9th September 2021; Published: $20^{\text {th }}$ September 2021

\section{Introduction}

Laboratory medicine has long become essential to medical care, with about $70 \%$ of medical decisions being influenced by in vitro diagnostic tests [1]. Due to its pivotal role, laboratory med- icine is also one of the fastest growing areas in medicine. With ever-improving techniques and technologies, new methods are introduced in clinical laboratories at an unprecedented rate. As modern automated laboratory analyzers are

\footnotetext{
* Corresponding author: Ion Bogdan Mănescu, Clinical Laboratory, County Emergency Clinical Hospital of Targu Mures, Romania. E-mail: manescu.bogdanion@gmail.com

\# These authors have contributed equally to this work and share first authorship
} 
generally faster and more precise, new methods have lower inherent error of measurement and have become increasingly more reliable and cost-effective. However, errors in the clinical laboratory are inevitable. Hence, it is the laboratory's responsibility to identify the manifold sources of error and address them according to national and international rules, regulations and quality standards such as described by the International Organization for Standardization (ISO) or by the Clinical Laboratory Standards Institute (CLSI), to name a few.

Replacing old methods with new ones is a natural and logical process for clinical laboratories aiming at improved performance and better patient care. However, a newly-introduced analyzer or method can itself be a source of error. Often, new analyzers must either run alongside with old ones or replace them altogether. In both cases, the laboratory must establish whether the two methods are comparable, that is, if the methods can be used interchangeably without adversely affecting medical decision-making and clinical outcome in patients. As comparability studies are frequently performed in clinical laboratories, many publications in scientific journals have approached the topic of method-comparison methodology [2]. Usually, statistical analysis of paired results from the two methods is needed, but the applicability of the new method must also be judged by considering the cost of a new analyzer, the cost and availability of reagents and calibration material, the space occupied by a new analyzer, operator education, waste handling, etc [2]. While decisions regarding applicability are based almost exclusively on local and subjective assessments, decisions concerning analytical performance usually depend on statistical analyses and objective criteria of acceptability [2].

The aim of this study was to determine the degree of comparability between the newly-introduced Atellica Solution CH 930 chemistry an- alyzer (Siemens AG, Germany) and the already established chemistry analyzers from our laboratory: Architect ci4100 (Abbott, US) and Cobas 6000 c501 (Roche, Switzerland).

\section{Material and Methods}

\section{Study design}

This study was performed between April and June 2020 in the Central Laboratory of Târgu Mureș County Emergency Clinical Hospital (Mureș County, Romania). Prior to this period, the operators of both the test and the comparative methods underwent a process of familiarization with the operation, maintainance procedures, calibration, function monitoring, and sample preparation of the new Atellica Solution CH 930 clinical chemistry analyzer. Daily quality control (QC) was performed for over 30 days before the study and throughout its entirety, using three levels of Bio-Rad controls (Ref 694, 695, 696; Lot 45830). Over the same period, calibration was performed regularly according to the manufacturer's instructions and occasionally if needed, using Atellica CH calibrators from Siemens Healthineers (Siemens AG, Germany). Data were not collected during the familiarization period.

According to EP09-A3 guideline [3], the comparative method can be the laboratory's current method, the method used by the manufacturer in the labeled claims, or a recognized reference method. In this study, the new Atellica Solution analyzer was compared with the laboratory's current method, that is with two chemistry analyzers already established in our laboratory: Cobas 6000 c501 and Architect ci4100, which are both part of a permanent proficiency testing/ external quality assessment program (PT/EQA). Therefore, throughout the study, the difference between the test and comparative methods is referred to simply as analytical difference $\left(D_{A}\right)$, and 
not bias. At the end of the comparative study, all 3 methods were tested against the same PT/EQA sample of unknown analyte concentrations.

\section{Sample collection and processing}

This study was performed on unhemolyzed patient samples which were randomly selected from routine testing at our laboratory and analyzed in singlicate directly from serum-separating tubes on both the test and comparative methods. For comparison of Atellica CH 930 with Architect ci4100, a short chemistry panel of 9 commonly investigated analytes was tested: alanine transaminase, aspartate aminotransferase, creatine kinase, creatinine, glucose, potassium, sodium, total bilirubin, and urea. For comparison of Atellica CH 930 with Cobas 6000 c501, an extended chemistry panel of 22 routine analytes was tested: albumin, alkaline phosphatase, alanine transaminase, amylase, aspartate aminotransferase, calcium, cholesterol, creatine kinase, creatinine, bilirubin (direct), gamma-glutamyl transferase, glucose, iron, lactate dehydrogenase, magnesium, potassium, sodium, bilirubin (total), protein (total), triglycerides, urea, and uric acid. Further information can be found in Table 1.

\section{Method comparison}

The Coefficient of analytical variation $\left(\mathrm{CV}_{A}\right)$ of the test method was calculated for all three control levels from daily QC data. Analytical difference $\left(D_{A}\right)$ between test and comparative methods was calculated at medical decision levels (MDL) [4] based on Passing-Bablok regression equa- tions. For enzymes, cholesterol, creatinine, triglycerides, urea, and uric acid, the lowest MDL [4] was excluded from analysis due to lack of clinical relevance. Total Error (TE) between test and comparative methods was calculated with the formula

$$
T E_{\text {obs }}=1.65 C V_{A}+D_{A}
$$

where $1.65 C V_{A}$ is the random error and $D_{A}$ is the systematic error. The acceptance limits for method comparison were based on three different criteria: analytical quality specifications derived from biological variation, CLIA proficiency testing criteria for acceptable analytical performance and SixSigma medical decision chart with $T E_{a}$ according to CLIA [5]. Data on biological variation were obtained from the Biological Variation Database of the European Federation of Clinical Chemistry and Laboratory Medicine (EFLM, https://biologicalvariation. $\mathrm{eu} /$ ) and, when not available, from the Biological Variation Database compiled by Dr. Carmen Ricos and colleagues [6]. Quality specifications derived from biological variation were calculated as shown below, using the internationally recognised analytical goals for imprecision and bias based on biological variation $[6,7]$. The tool for single-analyte Six Sigma evaluation was provided by www.westgard.com. Normalized method decision charts for multi-analyte Six Sigma evaluation (Figure 1) were generated using a Microsoft-Excel based tool created by the authors following a model published by Smolcic and Bilic-Zulle [8].

$$
\begin{array}{cccc}
\text { Optimum: } & C V_{A} \leq 0.25 \times C V_{I} & \text { Optimum: } & D_{A} \leq 0.125\left(C V_{I}^{2}+C V_{G}^{2}\right)^{1 / 2} \\
\text { Desirable: } & C V_{A} \leq 0.50 \times C V_{I} & \text { Desirable: } & D_{A} \leq 0.250\left(C V_{I}^{2}+C V_{G}^{2}\right)^{1 / 2} \\
\text { Minimum: } & C V_{A} \leq 0.75 \times C V_{I} & \text { Minimum: } & D_{A} \leq 0.375\left(C V_{I}^{2}+C V_{G}^{2}\right)^{1 / 2} \\
\text { Optimum: } & T E_{a} \leq 1.65\left(0.25 C V_{I}\right)+0.125\left(C V_{I}^{2}+C V_{G}^{2}\right)^{1 / 2} \\
\text { Desirable: } & T E_{a} \leq 1.65\left(0.50 C V_{I}\right)+0.250\left(C V_{I}^{2}+C V_{G}^{2}\right)^{1 / 2} \\
\text { Minimum: } & T E_{a} \leq 1.65\left(0.75 C V_{I}\right)+0.375\left(C V_{I}^{2}+C V_{G}^{2}\right)^{1 / 2}
\end{array}
$$


The equations above show the levels of analytical goals for imprecision $\left(\mathrm{CV}_{A}\right)$ and analytical difference $\left(D_{A}\right.$, substitute for bias) based on biological variation. If an agreement is desired between the test and comparative methods, both parameters must meet the specified performance criteria and can therefore be conveniently combined as $T E_{a}$ (Total Error allowable) for which three similar levels of analytical goals can be set.

$C V_{A}-$ coefficient of analytical variation (imprecision), $D_{A}$ - difference (systematic error, substitute for bias), $C V_{I}$ - coefficient of variation (intra-individual) derived from biological variation, $C V_{G}$ - coefficient of variation (inter-individual) derived from biological variation.

\section{Statistical processing}

All data were processed and organized using Microsoft Excel software (Microsoft Corporation, USA). Correlation, linear regression and Passing-Bablok regression statistical tests were performed on all data sets using MedCalc v14 software. The difference $\left(D_{A}\right)$ between methods was calculated at each MDL using Passing-Bablok regression equations, and further combined with the $C V_{A}$ of the test method in order to calculate the total error observed $\left(T E_{o b s}\right)$ between methods. SixSigma scores were calculated using a Microsoft Excel-based tool.

\section{Results}

All numerical data are presented in Tables 1-5. The following abbreviations will be used for the 22 analytes investigated in this study: Alb - albumin, ALP - alkaline phosphatase, ALT - alanine transaminase, Amy - amylase, AST - aspartate aminotransferase, $\mathrm{Ca}$ - calcium, Chol - cholesterol, CK - creatine kinase, Crea - creatinine, DBil - bilirubin (direct), GGT - gamma-glutamyl transferase, Gluc - glucose, LDH - lactate dehydrogenase, $\mathrm{Mg}$ - magnesium, $\mathrm{K}$ - potassi- um, Na - sodium, TBil - bilirubin (total), TProt - protein (total), Trig - triglycerides, UA - uric acid; Iron and Urea are not abbreviated.

\section{Discussions}

\section{Atellica Solution features}

The new Atellica Solution from Siemens Healthineers (Germany) was introduced in our laboratory at the end of the year 2019. This flexible and scalable system integrates high throughput immunoassay (IM 1300) and chemistry $(\mathrm{CH}$ 930) analyzers with a rapid bidirectional variable-speed magnetic sample transport line, multi-camera vision system with $360^{\circ}$ view, intelligent scheduling software, automated scheduling and delivery of controls and calibrators from an onboard refrigerated compartment, and other features designed for minimal operator intervention and turnaround times.

\section{Total analytical error and method comparison rationale}

As described above, the laboratory must determine whether the newly-introduced method is comparable with the current methods in our laboratory. Method comparison studies investigate total analytical error (TE) which is the summation of random error and systematic error. Random error is caused by variability in the operation of the method and does not relate to the true value, but is a matter of precision [2]. The counterpart of precision is imprecision, which is calculated as either standard deviation (SD) from the mean or a coefficient of variation (CV). Close monitoring of imprecision through QC procedures is important as it allows the examiner to determine whether changes in the value of a measurand can be explained by the inherent imprecision of the method alone, or other undesired factors should be considered and investigated. Systematic error, also known as bias or inaccuracy, is a measure of trueness, which is another essential 
Table 1. Samples used for method comparison. A variable number of samples was randomly picked each day. The duration of the comparison study is measured in days and can be different between analytes.

Total number of samples (n) is provided for each analyte as well as the lowest/highest recorded value and the median value.

\begin{tabular}{|c|c|c|c|c|c|c|}
\hline Assay & Units & Days & $\mathbf{n}$ & Min. value & Max. value & Median value \\
\hline \multicolumn{7}{|c|}{ Atellica CH 930 vs Architect ci4100 } \\
\hline ALT & $\mathrm{U} / \mathrm{L}$ & 11 & 245 & 6 & 3061 & 24 \\
\hline AST & $\mathrm{U} / \mathrm{L}$ & 11 & 291 & 8 & 3644 & 30 \\
\hline Crea & $\mathrm{mg} / \mathrm{dL}$ & 11 & 326 & 0.25 & 16.19 & 0.90 \\
\hline $\mathrm{CK}$ & $\mathrm{U} / \mathrm{L}$ & 14 & 111 & 15 & 8658 & 172 \\
\hline Gluc & $\mathrm{mg} / \mathrm{dL}$ & 11 & 337 & 43 & 510 & 113 \\
\hline $\mathrm{K}$ & $\mathrm{mmol} / \mathrm{L}$ & 12 & 219 & 2.15 & 6.68 & 4.06 \\
\hline $\mathrm{Na}$ & $\mathrm{mmol} / \mathrm{L}$ & 12 & 226 & 121 & 168 & 141 \\
\hline TBil & $\mathrm{mg} / \mathrm{dL}$ & 12 & 231 & 0.12 & 18.06 & 0.60 \\
\hline Urea & $\mathrm{mg} / \mathrm{dL}$ & 11 & 336 & 6.6 & 321.4 & 43.1 \\
\hline \multicolumn{7}{|c|}{ Atellica CH 930 vs Cobas 6000 c501 } \\
\hline Alb & $\mathrm{g} / \mathrm{dL}$ & 14 & 155 & 1.37 & 5.20 & 3.00 \\
\hline ALP & $\mathrm{U} / \mathrm{L}$ & 15 & 172 & 37 & 934 & 105 \\
\hline ALT & $\mathrm{U} / \mathrm{L}$ & 13 & 298 & 5 & 2145 & 28 \\
\hline Amy & $\mathrm{U} / \mathrm{L}$ & 13 & 162 & 9 & 1540 & 60 \\
\hline AST & $\mathrm{U} / \mathrm{L}$ & 13 & 312 & 7 & 866 & 35 \\
\hline $\mathrm{Ca}$ & $\mathrm{mmol} / \mathrm{L}$ & 21 & 154 & 1.03 & 4.10 & 2.23 \\
\hline Chol & $\mathrm{mg} / \mathrm{dL}$ & 15 & 178 & 45 & 369 & 173 \\
\hline CK & $\mathrm{U} / \mathrm{L}$ & 16 & 164 & 10 & 9123 & 79 \\
\hline Crea & $\mathrm{mg} / \mathrm{dL}$ & 13 & 301 & 0.09 & 8.61 & 0.82 \\
\hline DBil & $\mathrm{mg} / \mathrm{dL}$ & 11 & 182 & 0.05 & 19.90 & 0.54 \\
\hline GGT & $\mathrm{U} / \mathrm{L}$ & 14 & 157 & 7 & 1426 & 98 \\
\hline Gluc & $\mathrm{mg} / \mathrm{dL}$ & 13 & 318 & 38 & 428 & 103 \\
\hline Iron & $\mu \mathrm{mol} / \mathrm{L}$ & 20 & 154 & 0.4 & 55.1 & 9.5 \\
\hline LDH & $\mathrm{U} / \mathrm{L}$ & 6 & 129 & 38 & 5889 & 257 \\
\hline $\mathrm{Mg}$ & $\mathrm{mmol} / \mathrm{L}$ & 17 & 62 & 0.26 & 1.37 & 0.77 \\
\hline $\mathrm{K}$ & $\mathrm{mmol} / \mathrm{L}$ & 13 & 341 & 1.97 & 7.81 & 4.02 \\
\hline $\mathrm{Na}$ & $\mathrm{mmol} / \mathrm{L}$ & 13 & 338 & 118 & 182 & 140 \\
\hline TBil & $\mathrm{mg} / \mathrm{dL}$ & 13 & 168 & 0.10 & 25.60 & 0.98 \\
\hline TProt & $\mathrm{g} / \mathrm{dL}$ & 13 & 158 & 3.02 & 10.59 & 5.62 \\
\hline Trig & $\mathrm{mg} / \mathrm{dL}$ & 15 & 179 & 27.1 & 801.3 & 131.4 \\
\hline Urea & $\mathrm{mg} / \mathrm{dL}$ & 13 & 303 & 5.8 & 373.2 & 49.5 \\
\hline UA & $\mathrm{mg} / \mathrm{dL}$ & 15 & 160 & 1.5 & 23.9 & 5.6 \\
\hline
\end{tabular}

aspect of quality assurance. Accuracy is all the more important in clinical laboratories because significantly innacurate values no longer reflect the biological status of the patient and may adversely affect clinical decision. Since perfect methods of measurement do not exist, the best estimate of a true value is a value produced by a reference method [2]. However, according to EP09-A3 guideline [3], the comparative method can be not only a recognized reference method, but also the method used by the manufacturer in the labeled claims, or the laboratory's current 


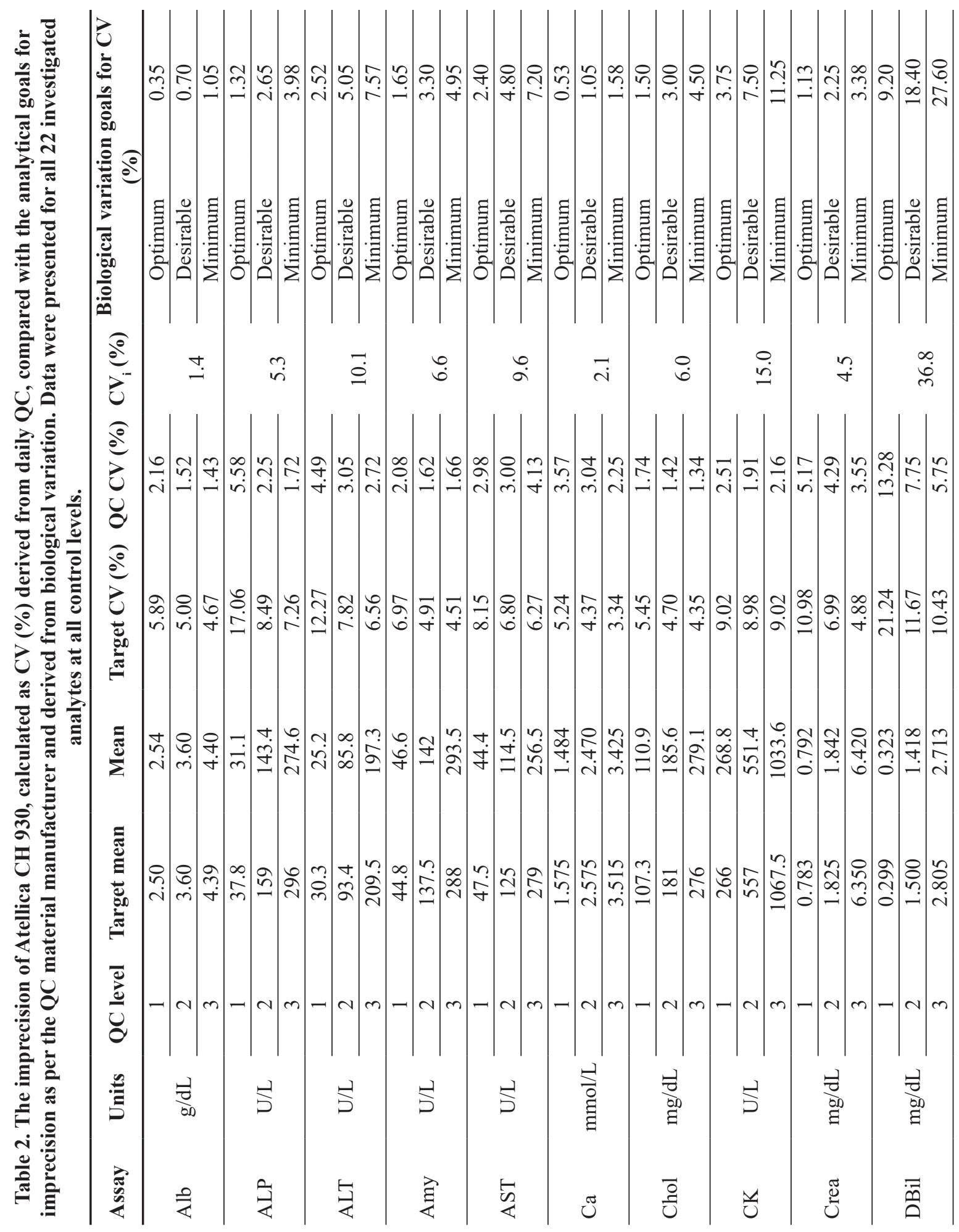




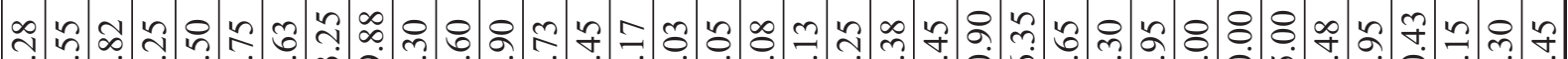
ते

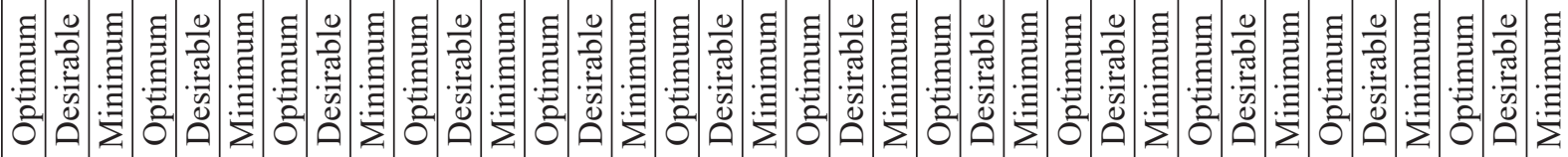

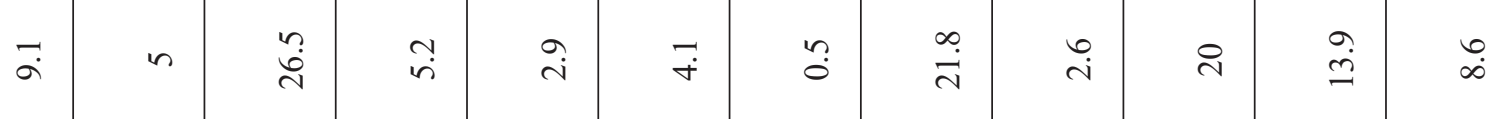

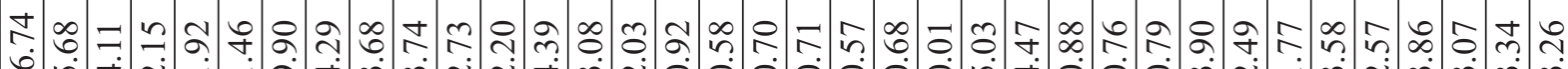

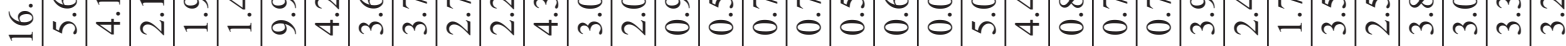

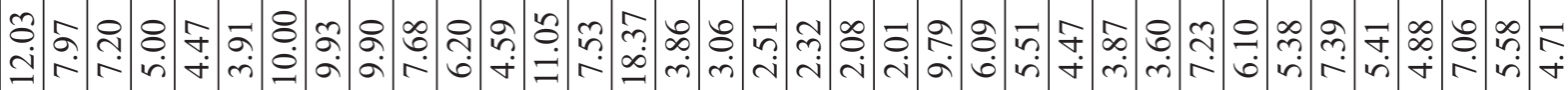

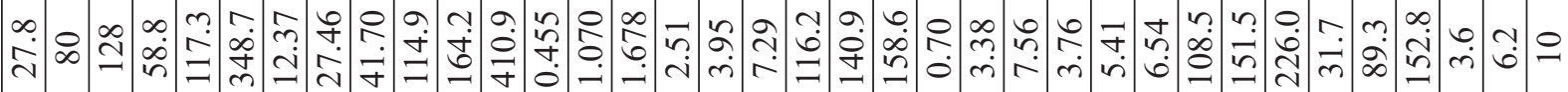

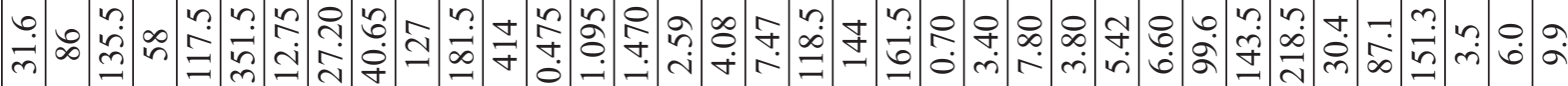

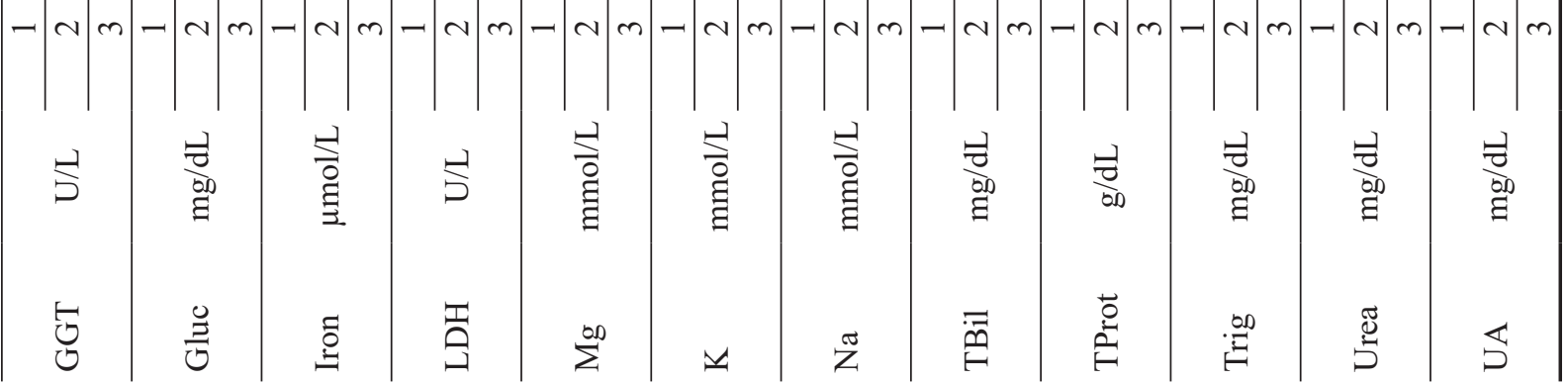


Table 3. Comparison of Atellica CH 930 (test method) and Architect ci4100 (comparative method) chemistry analyzers for 9 commonly investigated analytes. Six Sigma scores and $\mathrm{TE}_{\mathrm{obs}}$ were calculated at each medical decision level (MDL). $\mathrm{TE}_{\text {obs }}$ was compared with $\mathrm{TE}_{\mathrm{a}}$ as per CLIA and biological variation data.

\section{Atellica CH 930 vs Architect ci4100}

\begin{tabular}{|c|c|c|c|c|c|c|c|c|c|}
\hline \multicolumn{6}{|c|}{ Method comparison data } & \multicolumn{3}{|c|}{ Performance criteria } & \multirow{3}{*}{$\begin{array}{l}\text { Sigma } \\
\text { metric }\end{array}$} \\
\hline Assay & Units & MDL & $\begin{array}{l}C V_{A} \\
(\%) \\
\end{array}$ & $\begin{array}{c}D_{A} \\
(\%) \\
\end{array}$ & $\begin{array}{l}T E_{o b s} \\
(\%)\end{array}$ & $\begin{array}{r}\text { Biologica } \\
\qquad E_{a}\end{array}$ & ation & $\begin{array}{c}\text { CLIA } \\
T E_{a}(\%)\end{array}$ & \\
\hline \multirow{3}{*}{ ALT } & \multirow{3}{*}{$\mathrm{U} / \mathrm{L}$} & & & & & Optimum & 8 & \multirow{3}{*}{20} & \\
\hline & & 60 & 3.05 & 4.2 & 9.2 & Desirable & 16.1 & & 5.2 \\
\hline & & 300 & 2.72 & 7.2 & 11.7 & Minimum & 24.1 & & 4.7 \\
\hline \multirow{3}{*}{ AST } & \multirow{3}{*}{$\mathrm{U} / \mathrm{L}$} & & & & & Optimum & 6.8 & \multirow{3}{*}{20} & \\
\hline & & 60 & 2.98 & 8.8 & 13.7 & Desirable & 13.6 & & 3.8 \\
\hline & & 300 & 4.13 & 10.6 & 17.4 & Minimum & 20.5 & & 2.3 \\
\hline \multirow{3}{*}{$\mathrm{CK}$} & \multirow{3}{*}{$\mathrm{U} / \mathrm{L}$} & & & & & Optimum & 11.3 & \multirow{3}{*}{30} & \\
\hline & & 240 & 2.51 & 3.2 & 8 & Desirable & 22.6 & & $>6$ \\
\hline & & 1800 & 2.16 & 0.2 & 3.8 & Minimum & 33.8 & & $>6$ \\
\hline \multirow{3}{*}{ Crea } & \multirow{3}{*}{$\mathrm{mg} / \mathrm{dL}$} & & & & & Optimum & 3.7 & \multirow{3}{*}{15} & \\
\hline & & 1.6 & 4.29 & 1.4 & 8.5 & Desirable & 7.4 & & 3.2 \\
\hline & & 6 & 3.55 & 1.8 & 7.7 & Minimum & 11.1 & & 3.7 \\
\hline \multirow{3}{*}{ Gluc } & \multirow{3}{*}{$\mathrm{mg} / \mathrm{dL}$} & 45 & 2.15 & 1.2 & 4.8 & Optimum & 3.3 & \multirow{3}{*}{10} & 4.1 \\
\hline & & 120 & 1.92 & 1.4 & 4.6 & Desirable & 6.5 & & 4.5 \\
\hline & & 180 & 1.92 & 1.9 & 5.1 & Minimum & 9.8 & & 4.2 \\
\hline \multirow{3}{*}{$\mathrm{K}$} & \multirow{3}{*}{$\mathrm{mmol} / \mathrm{L}$} & 3 & 0.92 & 2.9 & 4.4 & Optimum & 2.4 & \multirow{3}{*}{6.7} & $>6$ \\
\hline & & 5.8 & 0.58 & 3.1 & 4 & Desirable & 4.8 & & $>6$ \\
\hline & & 7.5 & 0.70 & 3.1 & 4.3 & Minimum & 7.3 & & 5.1 \\
\hline \multirow{3}{*}{$\mathrm{Na}$} & \multirow{3}{*}{$\mathrm{mmol} / \mathrm{L}$} & 115 & 0.71 & 1.7 & 2.9 & Optimum & 0.3 & \multirow{3}{*}{2.7} & 2.5 \\
\hline & & 135 & 0.57 & 1.5 & 2.4 & Desirable & 0.7 & & 2.6 \\
\hline & & 150 & 0.57 & 1.3 & 2.3 & Minimum & 1 & & 2.4 \\
\hline \multirow{3}{*}{ TBil } & \multirow{3}{*}{$\mathrm{mg} / \mathrm{dL}$} & 1.4 & 0.10 & 8.6 & 8.8 & Optimum & 13.5 & \multirow{3}{*}{20} & $>6$ \\
\hline & & 2.5 & 5.03 & 9.2 & 17.5 & Desirable & 26.9 & & 2.1 \\
\hline & & 20 & 4.47 & 9.9 & 17.3 & Minimum & 40.4 & & 2.3 \\
\hline \multirow{3}{*}{ Urea } & \multirow{3}{*}{$\mathrm{mg} / \mathrm{dL}$} & & & & & Optimum & 8.9 & \multirow{3}{*}{9} & \\
\hline & & 56 & 3.58 & 7.7 & 13.6 & Desirable & 17.8 & & 0.4 \\
\hline & & 107 & 2.57 & 5.5 & 9.7 & Minimum & 26.6 & & 1.4 \\
\hline
\end{tabular}

method. For practical reasons, in this study the new Atellica Solution biochemistry analyzer $\mathrm{CH}$ 930 was compared with the laboratory's current validated methods: Architect ci4100 and Cobas 6000 c501.

Architect ci4100 is currently the biochemistry analyzer in our emergency department laboratory. Thus, the comparison between Architect ci4100 and Atellica $\mathrm{CH} 930$ was performed on a limited panel of 9 essential and commonly investigated analytes (see Table 1). In contrast, the comparison was performed on an extensive panel of 22 routine analytes for Cobas 6000 c501 (see Table 1), since this analyzer serves all clinical departments of our hospital and therefore accomodates a wider range of analytes. 


\section{Observed imprecision of Atellica $\mathrm{CH} 930$}

It must be noted that the precision of Atellica $\mathrm{CH}$ 930 chemistry analyzer, measured as CV derived from daily QC, was well within the allowed CV stated by the QC material manufacturer for all analytes, at all control levels (see Table 2), except for Iron (Control level 1: QC CV 9.9\% vs Target CV 10\%) and GGT (Control level 1: QC CV $16.74 \%$ vs Target CV 12.03\%). This is a welcome yet predictable finding as the imprecision figures for QC material are known to be often very wide [9]. On the other hand, analytical performance requirements based on biological variation $(\mathrm{BV})$ are considerably more demanding and therefore introduce a stricter, but more reliable set of analytical goals for imprecision. As presented in Table 2, Atellica CH 930 fulfilled or narrowly missed the BV analytical goals for optimum precision at all 3 control levels for 8 out of 22 investigated analytes: amylase, cholesterol, creatine kinase, potassium, total bilirubin, total protein, triglycerides, and urea. Various combinations of goals for optimum and desirable precision were achieved among the 3 control levels for each of the following 6 analytes: ALT, AST, direct bilirubin, glucose, iron, and uric acid. ALP, GGT, LDH showed a mixture of inacceptable/ minimum/ desirable precision, while inacceptable precision ( $\mathrm{CV}>\mathrm{CV}$ for minimum precision) was observed at all 3 control levels for albumin, calcium, creatinine, sodium, and at 2 out of 3 control levels for magnesium. Such diversity of results reflects the stark contrast between precision requirements of commercial QC material manufacturers and those derived from BV. Moreover, it shows that the expectation to always achieve in practice the optimum or desirable BV analytical goals, may sometimes be unrealistic [10].

\section{Observed vs allowable total analytical error}

Total error observed $\left(\mathrm{TE}_{\mathrm{obs}}\right)$ between the test and comparative methods was calculated at each medical decision level (MDL). The figures are presented in Tables 3 and 4 along with total allowable error (TE) limits as per CLIA and BV data. There are 3 levels of analytical goals for BV-TE : optimum (O), desirable (D), and minimum (M) [7]. TE obs that fails to meet the minimum specifications is considered inacceptable (F). While minimum specifications for BV- $\mathrm{TE}_{\mathrm{a}}$ may be too permissive, optimum BV- $\mathrm{TE}_{\mathrm{a}}$ may sometimes be too demanding and difficult to achieve in practice. Therefore, desirable $\mathrm{TE}_{\mathrm{a}}$ is the standard analytical goal in most laboratories that use performance specifications derived from BV [6].

As shown in Table 3, $\mathrm{TE}_{\text {obs }}$ between Atellica $\mathrm{CH}$ 930 and Architect ci4100 met or even exceeded the desirable analytical goals at all MDLs for 6 out of 9 analytes: ALT (2D-3D), creatine kinase (2O-3O), glucose (1D-2D-3D), potassium (1D-2D-3D), total bilirubin (1O-2D-3D), and urea (2D-3D). For AST (2D-3M), TE obs $_{\text {met the }}$ desirable analytical goal at just one MDL, while less than desirable performance at all 3 MDLs was observed for creatinine (2M-3M) and sodium (1F-2F-3F). Since inacceptable precision can easily determine inacceptable TE, failure to meet the desirable analytical goals for TE was expected for creatinine and sodium, given the inacceptable $\mathrm{CV}_{\mathrm{A}}$ recorded for these 2 analytes (see Table 2). Since the BV-derived analytical performance specifications (APS) are not well suited for all analytes [11], such "failures" should not always be judged too harshly. However, creatinine and sodium are among the analytes that have been proposed for the BV-derived APS [11] and we should therefore consider that the $\mathrm{TE}_{\mathrm{obs}}$ for these 2 analytes between Atellica CH 930 and Architect ci4100 is inacceptable.

Table 4 shows the comparison data for Atellica $\mathrm{CH} 930$ vs Cobas 6000 c501. TE obs $_{\text {met }}$ or even exceeded the BV desirable analytical goals at all 3 MDLs for 13 out of 22 analytes: ALP (2O$3 \mathrm{O})$, amylase (2D-3D), cholesterol (2D-3O), 


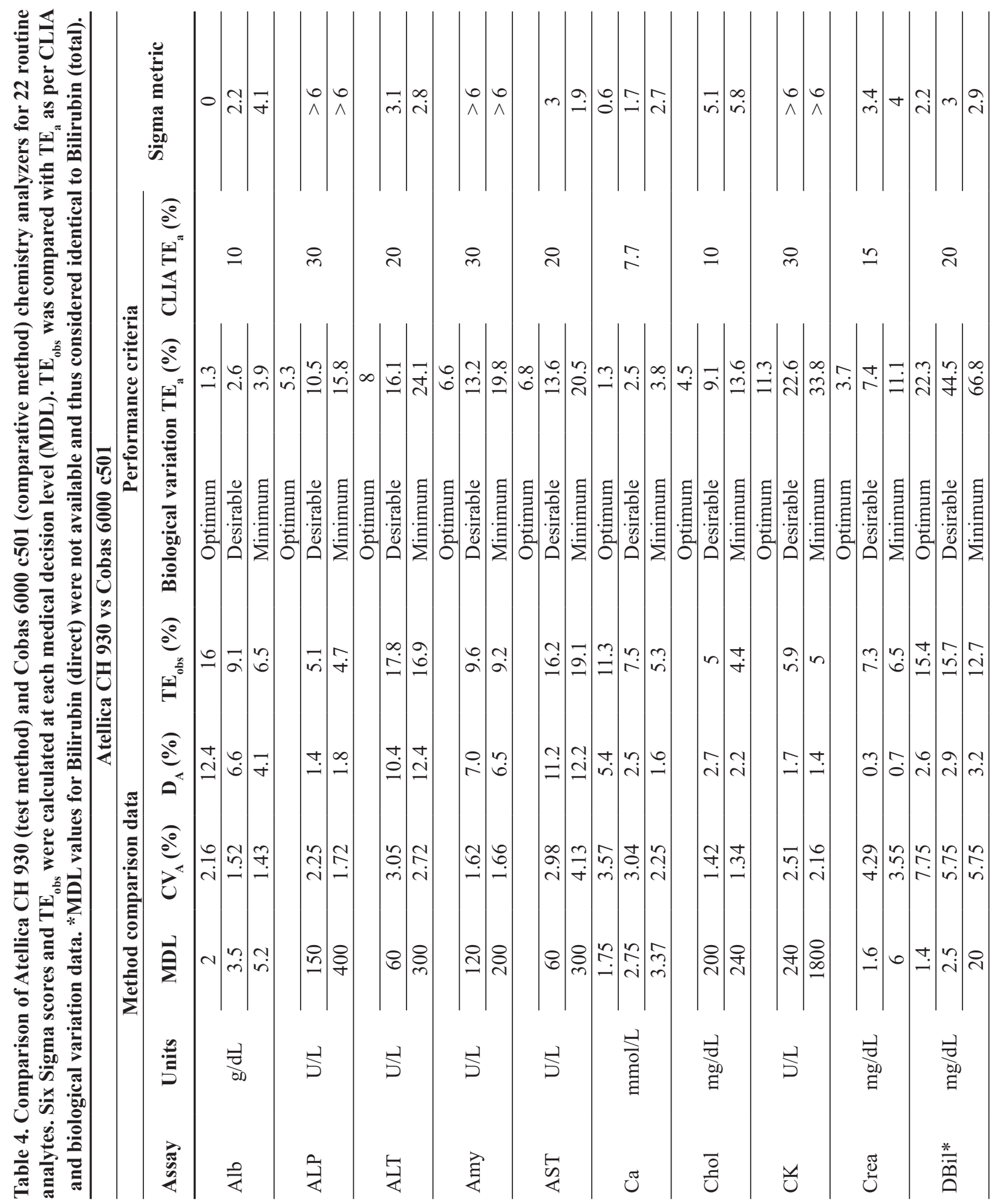




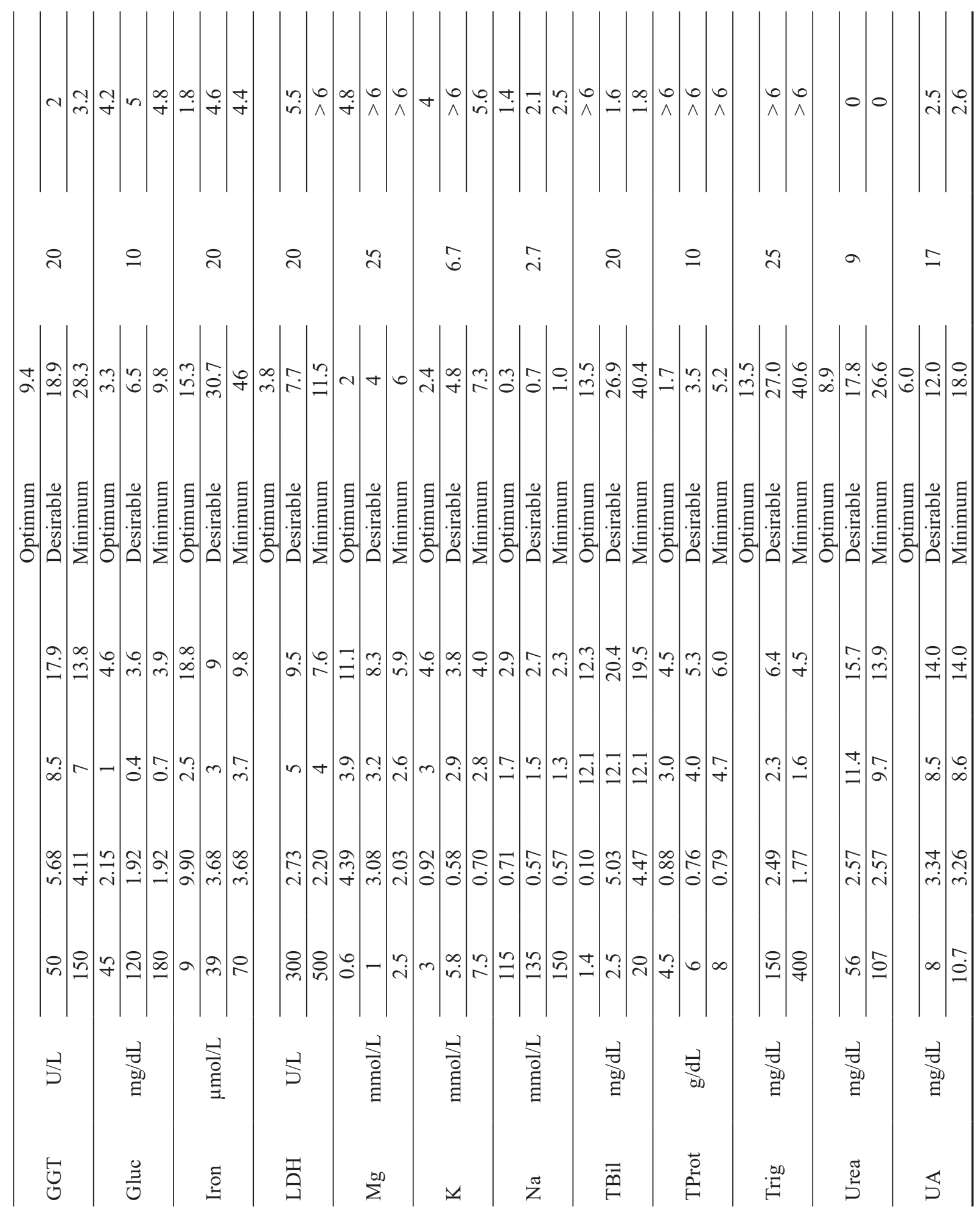


Table 5. PT/EQA results for the test method (Atellica CH 930) and both comparative methods (Architect ci4100 and Cobas $6000 \mathrm{c501}$ ). The test was not performed for amylase, but 4 additional analytes were investigated: chloride, high-density lipoprotein cholesterol (HDL-C), inorganic phosphate (IP), and low-density lipoprotein cholesterol (LDL-C).

\begin{tabular}{|c|c|c|c|c|c|c|c|c|c|}
\hline \multirow{2}{*}{ Assay } & \multirow{2}{*}{ Units } & \multicolumn{2}{|c|}{ EQA global results } & \multicolumn{2}{|c|}{ Architect ci4100 } & \multicolumn{2}{|c|}{ Atellica CH 930} & \multicolumn{2}{|c|}{ Cobas 6000 c501 } \\
\hline & & Target & SD & Result & Z score & Result & $\mathrm{Z}$ score & Result & $Z$ score \\
\hline Alb & $\mathrm{g} / \mathrm{dL}$ & 5.20 & 0.30 & & & 5.5 & 1.00 & 5.30 & 0.33 \\
\hline ALP & $\mathrm{U} / \mathrm{L}$ & 311.5 & 51.2 & & & 293 & -0.36 & 276 & -0.69 \\
\hline ALT & $\mathrm{U} / \mathrm{L}$ & 184 & 14.5 & 180 & -0.28 & 188 & 0.28 & 169.3 & -1.01 \\
\hline AST & $\mathrm{U} / \mathrm{L}$ & 173 & 11.9 & 167 & -0.51 & 183 & 0.84 & 165.9 & -0.60 \\
\hline $\mathrm{Ca}$ & $\mathrm{mmol} / \mathrm{L}$ & 3.38 & 0.17 & & & 3.54 & 0.94 & 3.49 & 0.60 \\
\hline Chloride & $\mathrm{mmol} / \mathrm{L}$ & 128 & 6.4 & 133 & 0.78 & 130 & 0.31 & & \\
\hline Chol & $\mathrm{mg} / \mathrm{dL}$ & 217 & 10.8 & & & 216 & -0.09 & 213.3 & -0.34 \\
\hline $\mathrm{CK}$ & $\mathrm{U} / \mathrm{L}$ & 288.1 & 30.3 & 288 & 0 & 279 & -0.30 & 295 & 0.23 \\
\hline Crea & $\mathrm{mg} / \mathrm{dL}$ & 3.57 & 0.33 & 4.07 & 1.52 & 4.12 & 1.66 & 3.59 & 0.06 \\
\hline DBil & $\mathrm{mg} / \mathrm{dL}$ & 1.32 & 0.21 & 1.41 & 0.43 & 1.50 & 0.86 & 1.29 & -0.14 \\
\hline GGT & $\mathrm{U} / \mathrm{L}$ & 88 & 4.5 & & & 78 & -2.22 & 87 & -0.22 \\
\hline Gluc & $\mathrm{mg} / \mathrm{dL}$ & 202 & 10.1 & 203 & 0.1 & 198.1 & -0.39 & 202 & 0 \\
\hline HDL-C & $\mathrm{mg} / \mathrm{dL}$ & 64 & 6.8 & & & 60.1 & -0.57 & 54.4 & -1.41 \\
\hline IP & $\mathrm{mmol} / \mathrm{L}$ & 1.97 & 0.11 & & & 2.12 & 1.36 & 1.98 & 0.09 \\
\hline Iron & $\mu \mathrm{mol} / \mathrm{L}$ & 51.76 & 2.59 & & & 53.07 & 0.50 & 50.52 & -0.48 \\
\hline $\mathrm{LDH}$ & $\mathrm{U} / \mathrm{L}$ & 343.5 & 17.2 & & & 341 & -0.14 & 348 & 0.26 \\
\hline LDL-C & $\mathrm{mg} / \mathrm{dL}$ & 136.3 & 9.9 & & & 142.1 & 0.58 & 129.7 & -0.67 \\
\hline $\mathrm{Mg}$ & $\mathrm{mmol} / \mathrm{L}$ & 1.40 & 0.08 & & & 1.45 & 0.63 & 1.42 & 0.26 \\
\hline $\mathrm{K}$ & $\mathrm{mmol} / \mathrm{L}$ & 5.87 & 0.34 & 5.80 & -0.21 & 5.68 & -0.56 & 5.82 & -0.15 \\
\hline $\mathrm{Na}$ & $\mathrm{mmol} / \mathrm{L}$ & 157.7 & 10.8 & 152 & -0.53 & 155 & -0.25 & 153 & -0.43 \\
\hline TBil & $\mathrm{mg} / \mathrm{dL}$ & 3.72 & 0.33 & 3.85 & 0.39 & 3.70 & -0.06 & 3.22 & -1.52 \\
\hline TProt & $\mathrm{g} / \mathrm{dL}$ & 8.30 & 0.42 & & & 7.92 & -0.90 & 8.04 & -0.62 \\
\hline Trig & $\mathrm{mg} / \mathrm{dL}$ & 182 & 9.1 & & & 194.2 & 1.34 & 187.5 & 0.60 \\
\hline Urea & $\mathrm{mg} / \mathrm{dL}$ & 87 & 4.6 & 85.4 & -0.35 & 92 & 1.08 & 86.3 & -0.15 \\
\hline UA & $\mathrm{mg} / \mathrm{dL}$ & 8.30 & 0.42 & & & 8.8 & 1.19 & 8.0 & -0.71 \\
\hline
\end{tabular}

creatine kinase (2O-3O), creatinine (2D-3D), direct bilirubin (1O-2O-3O), GGT (2D-3D), glucose (1D-2D-3D), iron (1D-2O-3O), potassium (1D-2D-3D), total bilirubin (1O-2D-3D), triglycerides (2O-3O), and urea (2D-3D). Good performance was observed also for ALT where $\mathrm{TE}_{\text {obs }}$ narrowly missed (*) the $\mathrm{BV}$ analytical goals for desirable TE at both MDLs (2M*$\left.3 \mathrm{M}^{*}\right)$. Lesser and/or mixed performance among MDLs was observed for the following 3 analytes: AST (2M-3M), LDH (2M-3D), and uric acid (2M-3M). Since AST and uric acid both have satisfactory $\mathrm{CV}_{\mathrm{A}}$ (random error), their performance was affected by a high $\mathrm{D}_{\mathrm{A}}$ (systematic error) caused by high slope values: AST ( $\mathrm{y}=$ $-0.76+1.125 \mathrm{x})$ and uric acid $(\mathrm{y}=-0.04+1.09 \mathrm{x})$. Finally, inacceptable performance was observed for albumin $(1 \mathrm{~F}-2 \mathrm{~F}-3 \mathrm{~F})$, calcium $(1 \mathrm{~F}-2 \mathrm{~F}-3 \mathrm{~F})$, magnesium $(1 \mathrm{~F}-2 \mathrm{~F}-3 \mathrm{M})$, sodium $(1 \mathrm{~F}-2 \mathrm{~F}-3 \mathrm{~F})$, and total protein $\left(1 \mathrm{M}-2 \mathrm{~F}^{*}-3 \mathrm{~F}\right)$, mainly due to the highly demanding analytical goals caused by low BV. However, given that calcium, magnesium, sodium, and total protein have been proposed for the BV-derived APS [11], we should 
consider that the $\mathrm{TE}_{\text {obs }}$ for these 4 analytes between Atellica CH 930 and Cobas 6000 c501 is inacceptable.

In clear contrast with desirable analytical goals derived from BV data, CLIA's requirements for analytical performance are generally less demanding (see Table 4, BV-TE vs CLIA-TE ). Thus, the performance of Atellica CH 930 compared to Architect ci4100 achieved or narrowly missed CLIA's requirements for all analytes at all MDLs (see Table 3), except for urea (all MDLs). However, being an analyte with high biological variation, urea is one of those notable exceptions where CLIA-TE $\mathrm{a}_{\mathrm{a}}$ is uncharacteristically more stringent than BV-TE, along with direct bilirubin, GGT, iron, total bilirubin, and triglycerides. In a similar fashion, $\mathrm{TE}_{\mathrm{obs}}$ between Atellica CH 930 and Cobas 6000 c501 achieved or narrowly missed CLIA-TE $\mathrm{a}_{\mathrm{a}}$ requirements for all analytes at all MDLs (see Table 4) except for albumin (MDL 1), calcium (MDL 1), and urea (all MDLs). CLIA's less stringent requirements are all the more evident when looking at analytes with low BV where $\mathrm{TE}_{\text {obs }}$ was inacceptable as per $\mathrm{BV}-\mathrm{TE}_{\mathrm{a}}$, but acceptable according to CLIA-TE (albumin, calcium, creatinine, magnesium, sodium, total protein). The popularity of both CLIA and BV-derived sets of APS, along with the evident contrast between the two, raise questions about the differences in method performance assessment and limits of acceptability across laboratories [12].

\section{Sigma metrics for assessment of laboratory analytical performance}

For the laboratory, it is important to avoid outliers and false positive/negative results. These erroneous results are the manifestation of the laboratory's total analytical error and can be viewed as "defects" generated by the measurement process. Sigma metrics is a technique to quantify (and then minimize) defects, that originated in the manufacturing industry and was first used in medical laboratories in the year 2000 [10]. The "Sigma" in Six Sigma refers to the benchmarking scale upon which all process defects are judged $(\sigma)$, while the "Six" refers to the ideal performance where the defined acceptable limits of a process can fit six standard deviations [10]. The sigma metric (SM) is calculated using the equation $\mathrm{SM}=\left(\mathrm{TE}_{\mathrm{a}} \%\right.$ - bias $\left.\%\right) / \mathrm{CV} \%$ and it can be used to calculate the number of defects per million opportunities (DPMO). For instance, $6 \sigma$ corresponds to 3.4 DPMO (99.99966\% yield), $3 \sigma$ to 66,807 DPMO (93.3\% yield), and $1 \sigma$ to 691,462 DPMO (30.9\% yield) [10]. Thus, most clinical laboratories using sigma metrics require that a minimum of $3 \sigma$ be achieved. Six Sigma has proved to be a comprehensible, useful and easy-to-use tool for visualization and tracking of analytical performance in the laboratory [10] and for method comparison studies [13]. Moreoever, the simplicity of assessing overall analytical performance with a single, adimensional metric, makes Six Sigma a great benchmarking tool and therefore a good candidate for the worldwide harmonization of clinical laboratories APS. Nevertheless, standing in the way of harmonization is the $\mathrm{TE}_{\mathrm{a}}$ component of the SM equation, which greatly varies between laboratories and especially across different countries [10]. As already mentioned, laboratories employ various analytical goals for $\mathrm{TE}_{\mathrm{a}}[10,12]$, the most popular being those derived from $\mathrm{BV}$ data or issued by relevant organizations such as CLIA, the College of American Pathologists (CAP), the German medical association for the quality assurance of laboratory medical examinations (RiliB̈̈K), and the Royal College of Pathologists of Australasia (RCPA). In this study, bias was replaced with the $\mathrm{D}_{\mathrm{A}}$ between the test and comparative methods and sigma metrics were computed at all MDLs using CLIA-TE .

The SMs computed for Atellica CH 930 vs Architect ci4100 at all 3 MDLs were presented in Table 3. SM values above 3 were observed for 
the following analytes: ALT, creatine kinase, creatinine, glucose, and potassium. For AST, SM values below 3 were observed at only one MDL. Sodium and total bilirubin showed lesser performance, with SM values between 2 and 3. Low SM values, between 0 and 2, were observed for urea. For comparison of Atellica $\mathrm{CH} 930$ with Cobas 6000 c501, SMs are shown in Table 4. SM values above 3 were observed at all MDLs for 11 analytes: ALP, amylase, cholesterol, creatine kinase, creatinine, glucose, LDH, magnesium, potassium, total protein, and triglycerides. SM values below 3 were observed at just one MDL for ALT, AST, direct bilirubin, GGT, and iron. SM values lower than 3 at multiple MDLs were observed for the other analytes, with Urea again showing the lowest performance $(0 \sigma$ at all MDLs). However, Six Sigma performance depends on the chosen $\mathrm{TE}_{\mathrm{a}}$ value $[10,13]$, and the results would be quite different for some analytes if BV-TE aere to be used instead of CLIA$\mathrm{TE}_{\mathrm{a}}$. Figure 1 was generated in order to better visualize the differences in Six Sigma analytical performance between choosing either BV-TE or CLIA-TE as the acceptable limits of the measurement process. With only a demonstrative purpose, Figure 1 exclusively shows the charts of Atellica CH 930 vs Cobas 6000 c501 as this comparison covers a wider range of analytes. The type of chart shown in Figure 1 (normalized MEDx chart - normalized method decision chart) enables the simultaneous presentation of multiple methods on the same chart. Performance parameters (imprecision/oX and inaccuracy/oY) are expressed relatively (normalized), as a percentage of $\mathrm{TE}_{\mathrm{a}}$.

A recent performance evaluation of Atellica $\mathrm{CH}$ 930 chemistry analyzer was performed at 4 different laboratories across Europe for 13 chemistry analytes [14]. In this study, Atellica CH 930 was compared with the ADVIA XPT systems (one site) and with the ADVIA 1800 systems (three sites) [14]. This robust multicentric study reported that, at the individual-site level, $90 \%$ of chemistry assays performed at 4 Sigma or high-

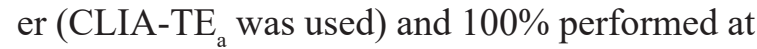
3 Sigma or higher, thus concluding in favor of the new Atellica $\mathrm{CH} 930$ analyzer and its use in the clinical laboratory [14]. In our study, when compared with Architect ci4100, Atellica CH 930 reached a 4 Sigma performance or higher at $50 \%$ of the investigated MDLs and a 3 Sigma performance or higher at $64 \%$ of MDLs (see Table 3). Similarly, when compared with Cobas 6000 c501, Atellica CH 930 reached a 4 Sigma performance or higher at $54 \%$ of the investigated MDLs and a 3 Sigma performance or higher at $63 \%$ of MDLs (see Table 4 ).

\section{Analytical total allowable error vs medically allowable error}

The practice of establishing APS based on pure analytical rationale and laboratory-centered views is flawed and has its limitations. In contrast, medically allowable error is patient-centered and focuses on clinical outcome. This concept arised in the second half of the 20th century and saw notable contributions from Skendzel, Barnett, and Platt [15]. However, the isolated efforts of several working groups failed to establish a reliable set of medically allowable errors and the little data available were obtained by various methodologies and are rather based on experts' opinion.

While defining the three models for APS derivation, the 2014 Milan consensus placed the model based on the effect of analytical performance on the clinical outcome, at the top of the hierarchy, thus reaffirming the superiority of this patient-centered view of analytical performance in the clinical laboratory [16]. Nevertheless, the lack of an extensive evidence-based database of medically allowable errors, remains a challenge to this day. Such an undertaking would require titanic interdisciplinary efforts starting with clinicians agreeing on medically allowable errors 

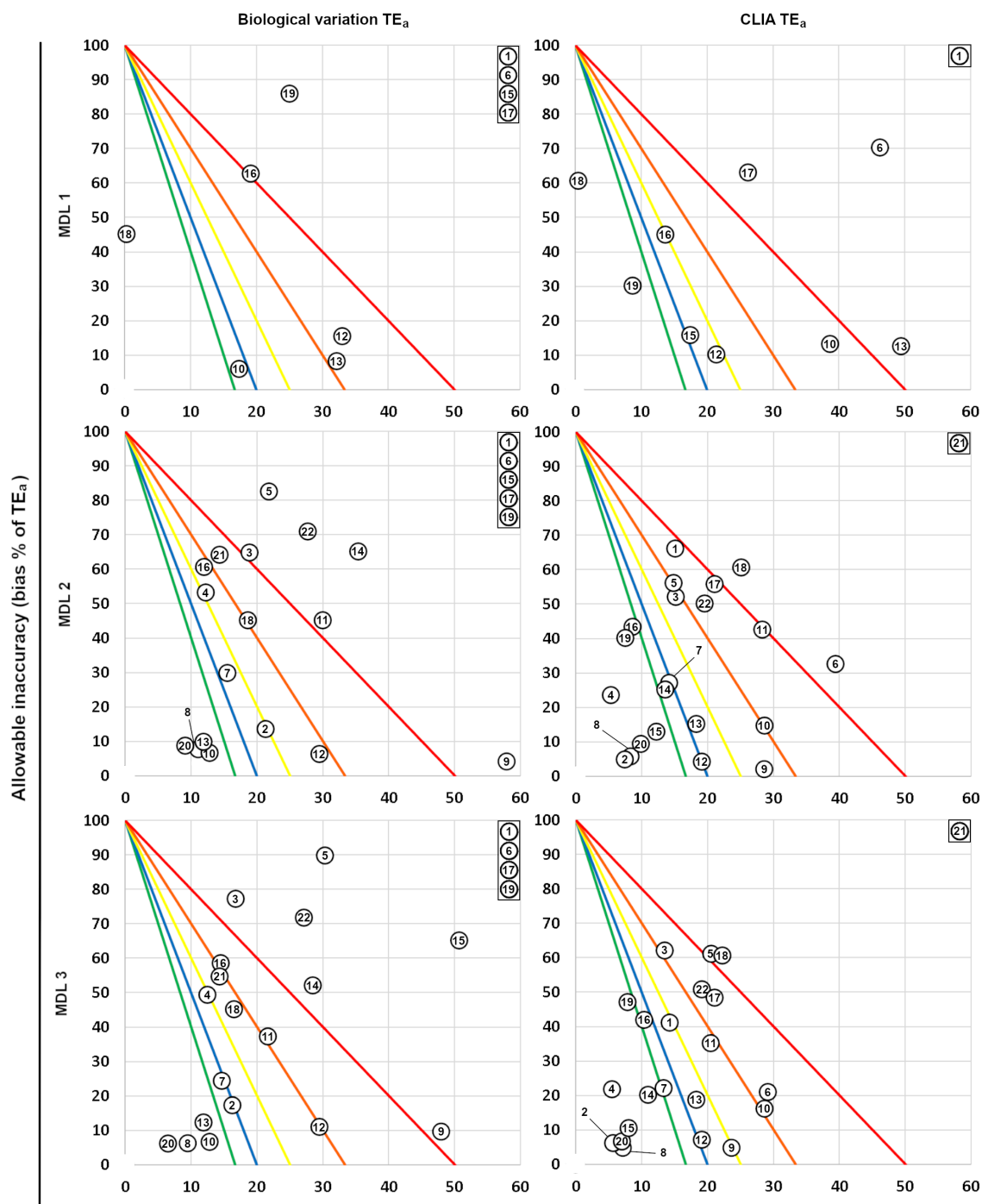

Allowable imprecision (CV \% of $\left.\mathrm{TE}_{\mathrm{a}}\right)$

World class

Excellent

Good Marginal

Poor

Figure 1. Normalized method decision charts showing Six Sigma performance of the test method (Atellica CH 930) compared with the comparative method (Cobas 6000 c501). In order to visualize the effect of different $\mathrm{TE}_{\mathrm{a}}$ values on sigma metrics, charts were generated for each medical decision level (MDL) with either biological variation $\mathrm{TE}_{\mathrm{a}}$ or CLIA $\mathrm{TE}_{\mathrm{a}}$ as analytical performance limits. Each investigated analyte is represented by a number in a circle. The cassette in the upper-right corner of each graph holds analytes that could not be plotted due to highly inacceptable Six Sigma performances. For enzymes, cholesterol, creatinine, triglycerides, urea, and uric acid, MDL1 was excluded from analysis due to lack of clinical relevance. 1 - Albumin, 2 Alkaline phosphatase, 3 -Alanine transaminase, 4 - Amylase, 5 - Aspartate aminotransferase, 6 - Calcium, 7 Cholesterol (total), 8 - Creatine kinase, 9 - Creatinine, 10 - Bilirubin (direct), 11 - gamma-glutamyl transferase, 12 - Glucose, 13 - Iron, 14 - Lactate dehydrogenase, 15 - Magnesium, 16 - Potassium, 17 - Sodium, 18 Bilirubin (total), 19 - Protein (total), 20 - Triglycerides, 21 - Urea, 22 - Uric acid. 
at multiple MDLs, with respect to the inherent particularities of each pathology, medical speciality, and clinical status of the patient. The first steps toward this goal were made for several analytes such as Total/ HDL/ LDL cholesterol, glucose, glycated hemoglobin, albumin, C-reactive protein, cardiac troponins, and hemoglobin [11]. Due to their central and well-defined roles in the decision making of a specific disease or clinical situation, these analytes have been the subject of extensive studies in order to establish cut-off values and multiple decision thresholds for the diagnosis, risk assessment and management of disease [11]. For the same reason, these analytes have already been proposed as candidates for the APS model based on clincal outcome [11]. Hopefully, with the implementation of more evidence-based and universally-accepted cut-off and threshold values, additional steps will also be made toward establishing the medically allowable error at each one of them.

\section{Test and comparative methods vs PT/EQA}

All numerical data presented in this study resulted from the comparison of the test method (Atellica CH 930) with the comparative methods (Architect ci4100 and Cobas 6000 c501). The level of agreement between the 3 methods should be acceptable in order for the laboratory to use them interchangeably. Although both comparative methods have already been validated in our laboratory and are part of a permanent PT/EQA program, the method comparison methodology chosen here has one notable limitation, that is, the inaccuracy of the test method remains unknown. Therefore, Atellica $\mathrm{CH} 930$ was enrolled for a one-time participation in the PT/ EQA program, along with the two comparative methods (see Table 5). All 3 chemistry analyzers performed well, with $\mathrm{Z}$ scores between -2 and 2 for all investigated analytes, except for Atellica's GGT which we have already reported as being problematic throughout the study. Moreover, apart from GGT, there was no investigated analyte where the difference between Atellica's Z score and the $\mathrm{Z}$ score of any of the two comparative methods, was greater than 2 . While for the uneducated eye these results may seem apparent proof of agreement between methods, one should not jump to conclusions, as these satisfactory PT/EQA results do not necessarily imply that the test and comparative methods are comparable and could be used interchangeably. This is because in a PT/EQA, the analytical performance acceptance limits are defined by a single metric, that is the $\mathrm{Z}$ score, whose calculus formula is so that an important inaccuracy can be obscured by a high SD/CV. This heavy reliance of $\mathrm{Z}$ score on $\mathrm{SD} / \mathrm{CV}$ is the more relevant since high SDs/CVs generally occur in such PT/EQA global results, due to the heterogeneity of the participants. For instance, the $\mathrm{CV}_{\mathrm{I}}$ for sodium is $0.5 \%$, which means the desirable $\mathrm{CV}_{\mathrm{A}}$ of the method should be less than $0.25 \%$. In stark contrast, the global results of the above-mentioned PT/EQA show a target value of $157.7 \mathrm{mmol} / \mathrm{L}$ and a SD of $10.8 \mathrm{mmol} / \mathrm{L}$ (see Table 5), that is a CV of $6.87 \%$. Therefore, while the limits of acceptance for the measurement process based on biological variation data are computed using a CV of $0.25 \%$, the analytical performance in a $\mathrm{PT} / \mathrm{EQA}$ is computed using a CV of $6.87 \%$. This is why Atellica's sodium method was proven not comparable with either comparative method (see Tables 2 and 3), while all 3 analyzers performed well in the PT/EQA, with similar Z scores (see Table 5, sodium). In short, heterogeneity among methods is expected in PTs/EQAs, but should not be tolerated within a laboratory with multiple analyzers, at least not when the laboratory's methods are used interchangeably/ alternatively for patient evaluation.

\section{Conclusions}

Performance of the newly introduced Atellica 
$\mathrm{CH}$ 930, as compared with the already established Architect ci4100 was acceptable or nearly acceptable at $82 \%, 95 \%$, and $64 \%$ of the 22 investigated MDLs across 9 analytes, according to BV-TE, CLIA-TE and CLIA-calculated Sigma metrics, respectively. Similarly, performance of the newly introduced Atellica $\mathrm{CH}$ 930, as compared with the already established Cobas 6000 c501 was acceptable or nearly acceptable at $61 \%, 93 \%$, and $63 \%$ of the 54 investigated MDLs across 22 analytes, according to BV-TE, CLIA-TE and CLIA-calculated Sigma metrics, respectively. However, method comparability should not be evaluated by a "one size fits all" approach as some analytes require different criteria of acceptability, ideally based on medically allowable error and clinical outcome.

There are several models for method comparison studies, all having their strengths and weaknesses. Also, despite recent steps toward standardization of analytical performance goals in the clinical laboratory, various schools of thought remain highly influential among laboratory professionals. These, along with the great variability of quality specifications required by different countries, regulatory agencies or PT/EQA providers, represent an important barrier in the way of harmonization, and significantly contribute to the perpetual lack of regard for evidence-based and patient-centered practices in the clinical laboratory.
Abbreviations
Alb - albumin
ALP - alkaline phosphatase
ALT - alanine transaminase
Amy - amylase
APS - analytical performance specifications
AST - aspartate aminotransferase
$\mathrm{BV}$ - biological variation
$\mathrm{Ca}$ - calcium
CAP - the College of American Pathologists
Chol - cholesterol

CK - creatine kinase

CLIA - Clinical Laboratory Standards Institute

Crea - creatinine

$\mathrm{CV}$ - coefficient of variation

$\mathrm{CV}_{\mathrm{A}}$ - coefficient of analytical variation

$\mathrm{CV}_{\mathrm{G}}$ - coefficient of inter-individual biological variation

$\mathrm{CV}_{\mathrm{I}}$ - coefficient of intra-individual biological variation

D - desirable

$\mathrm{D}_{\mathrm{A}}$ - analytical difference between methods (substitute for bias)

DBil - bilirubin (direct)

DPMO - defects per million opportunities

EFLM - European Federation of Clinical Chemistry and Laboratory Medicine

EQA - external quality assessment program

F - inacceptable

GGT - gamma-glutamyl transferase

Gluc - glucose

HDL-C - high-density lipoprotein cholesterol

IP - inorganic phosphate

ISO - International Organization for Standardization

$\mathrm{K}$ - potassium

$\mathrm{LDH}$ - lactate dehydrogenase

LDL-C - low-density lipoprotein cholesterol

$\mathrm{M}-$ minimum

MDL - medical decision level

MEDx chart - method decision chart

$\mathrm{Mg}$ - magnesium

$\mathrm{Na}$ - sodium

$\mathrm{O}$ - optimum

PT - proficiency testing

QC - quality control

RCPA - the Royal College of Pathologists of Australasia

RiliBÄK - the German medical association for the quality assurance of laboratory medical examinations

SD - standard deviation

SM - sigma metric

TBil - bilirubin (total)

$\mathrm{TE}$ - total error 
$\mathrm{TE}_{\mathrm{a}}$ - total error allowable

$\mathrm{TE}_{\mathrm{obs}}$ - total error observed between methods

TProt - protein (total)

Trig - triglycerides

$\mathrm{UA}$ - uric acid

\section{Acknowledgements}

We would like to thank our colleagues Andreea Truță and Floredana Șular for their technical assistance with the Atellica CH 930 chemistry analyzer. This study was supported by Sante International SA, the official distributor in Romania for Siemens IVD products.

\section{Authors' contributions}

IG, KP, IBM: conceptualization, investigation, methodology, validation, visualization, writing (original draft preparation).

ORO: conceptualization, methodology, validation, supervision, writing (original draft preparation).

MD: conceptualization, methodology, resources, supervision, writing (review and editing).

\section{Conflict of interest}

The authors declare that there is no conflict of interest.

\section{References}

1. Rohr UP, Binder C, Dieterle T, Giusti F, Messina $\mathrm{CG}$, Toerien E, et al. The value of in vitro diagnostic testing in medical practice: a status report. PLoS One. 2016;11:e0149856 DOI: 10.1371/journal. pone. 0149856

2.Jensen AL, Kjelgaard-Hansen M. Method comparison in the clinical laboratory. Vet Clin Pathol. 2006 Sep;35(3):276-86. DOI: 10.1111/j.1939-165X.2006. tb00131.x

3. Clinical and Laboratory Standards Institute. Measurement Procedure Comparison and Bias Estimation Using Patient Samples; Approved guideline - Third Edition. Wayne, PA: Clinical and Laboratory Standards Institute, 2013. CLSI Document EP09-A3.

4. Statland BE. Clinical Decision Levels for Laboratory Tests, Second Edition. Oradell NJ, Medical Economics
Books, 1987.

5. U.S. Centers for Medicare \& Medicaid Services (CMS). Medicare, Medicaid, and CLIA programs: laboratory requirements relating to quality systems and certain personnel qualifications. Final Rule. Fed Regist 2003; 16:3650-714.

6. Ricos C, Alvarez V, Cava F, Garcia-Lario JV, HernandezA, Jimenez CV, et al. Current databases on biological variation: pros, cons and progress. Scand J Clin Lab Invest. 1999;59:491-500 DOI: 10.1080/00365519950185229

7. White GH, Farrance I; AACB Uncertainty of Measurement Working Group. Uncertainty of measurement in quantitative medical testing: a laboratory implementation guide. Clin Biochem Rev. 2004;25(4):S1-S24.

8. Smolcic VS, Bilic-Zulle L. Normalized MEDx chart as a useful tool for evaluation of analytical quality achievements. A picture is worth a thousand words. Clin Chem Lab Med. 2013;51(5):e99-e101. DOI: 10.1515/cclm2012-0629

9. Sikaris K. Analytical quality - what should we be aiming for?. Clin Biochem Rev. 2008;29(Suppl 1):S5-S10.

10. Westgard S, Bayat H, Westgard JO. Analytical Sigma metrics: A review of Six Sigma implementation tools for medical laboratories. Biochem Med (Zagreb). 2018;28(2):020502. DOI: 10.11613/BM.2018.020502

11. Ceriotti F, Fernandez-Calle P, Klee GG, Nordin G, Sandberg S, Streichert T, et al. Criteria for assigning laboratory measurands to models for analytical performance specifications defined in the 1st EFLM Strategic Conference. Clin Chem Lab Med. 2017 Feb;55(2):189194. DOI: $10.1515 / \mathrm{cclm}-2016-0091$

12. Friedecky B, Kratochvila J, Budina M. Why do different EQA schemes have apparently different limits of acceptability? Clin Chem Lab Med. 2011 Apr;49(4):7435. DOI: 10.1515/CCLM.2011.105

13. Hens K, Berth M, Armbruster D, Westgard S. Sigma metrics used to assess analytical quality of clinical chemistry assays: importance of the allowable total error (TEa) target. Clin Chem Lab Med. 2014 Jul;52(7):973-80. DOI: 10.1515/cclm-2013-1090

14. Fasano T, Bedini JL, Fle PA, Jlaiel M, Hubbert K, Datta $\mathrm{H}$, et al. Multi-site performance evaluation and Sigma metrics of 20 assays on the Atellica chemistry and immunoassay analyzers. Clin Chem Lab Med. 2019 Dec;58(1):59-68. DOI: 10.1515/cclm-2019-0699

15. Skendzel LP, Barnett RN, Platt R. Medically useful criteria for analytic performance of laboratory tests. Am J Clin Pathol. 1985 Feb;83(2):200-5. DOI: 10.1093/ ajcp/83.2.200

16. Sandberg S, Fraser CG, Horvath AR, Jansen R, Jones $\mathrm{G}$, Oosterhuis W, et al. Defining analytical performance specifications: Consensus Statement from the 1st Strategic Conference of the European Federation of Clinical Chemistry and Laboratory Medicine. Clin Chem Lab Med. 2015 May;53(6):833-5. DOI: 10.1515/cclm2015-0067 